\title{
System condition monitoring through Bayesian change point detection using pump vibrations*
}

\author{
E. Tochev, D. Rengasamy, H. Pfifer, S. Ratchev
}

\begin{abstract}
This paper presents a method for vibration analysis and a simple test bench analogue for the solder pumping system in an industrial wave-soldering machine at a Siemens factory. A common machine fault is caused by solder build-up within the pipes of the machine. This leads to a pressure drop in the system, which is replicated in the test bench by restricting the flow of water through the use of a gate valve. The pump's vibrational response is recorded using an accelerometer. The captured data is passed through a Bayesian Change point Detection algorithm, to detect the point at which the change in flow rate affects the pump, and thus the machine output. This information can be used to isolate the vibrational response indicative of the machine fault, which can then inform maintenance procedures.
\end{abstract}

\section{INTRODUCTION}

This paper describes a non-disruptive technique to detect a machine fault in a wave-soldering machine in PCB manufacturing. The fault in question is solder build-up in the machine pipes, which was considered the most common recurring machine fault in the wave-soldering process by Siemens Congleton. The system maintains a constant flow of solder in order to provide a set wave height for PCB soldering. Over time, solder dross builds up in the pipes and results in reduced wave height, leading to unsoldered products that require repairs or touch-ups. Once this is detected, pump power is manually increased to raise the wave height, compensating for the reduced pressure. After the power is raised over a threshold, the machine is turned off and maintenance is performed. This is a reactive process repeated on a weekly to monthly basis, depending on the length of machine uptime and quality of solder. The project brief set by Siemens requires a non-disruptive and low-cost solution. The goal is to improve the fault detection rate and reduce the number of unsoldered products.

Knowledge about the machine state can feed into predictive maintenance decisions. Early and accurate fault detection is key to minimising machine downtime and overall maintenance costs [1]. Additional value has been added by the current industrial push towards I4.0 and connected factories: using an Industrial Internet of Things (IIoTs) allows for greater abilities to gather and process machine data [2]. Despite this, the time and cost of replacing machines can dissuade companies from upgrading. Instead, retrofitting and a "wrap and re-use" attitude is considered preferable [3]. This is a key motivator for this paper and the proposed

*Research supported by the EPSRC and Siemens Congleton.

E. Tochev, D. Rengasamy, H. Pfifer, S. Ratchev are with the Institute for Advanced Manufacture, University of Nottingham, UK. E. Tochev is the corresponding author (e-mail: emil.tochev@nottingham.ac.uk). methodology - the minimal disruption to existing machines and processes allows for the extraction of new data from the available resources, without any requirement for machine downtime.

Pump vibrations are used for the vibration analysis in this paper because other data cannot realistically be collected from the wave-soldering machine non-disruptively. Pump vibration monitoring is not unique, and many examples can be found in literature [4], [5]. Techniques include comparing recorded pump vibrations against a model of the expected vibrations [6] and using pump vibrations to predict cavitation by training an Artificial Neural Network (ANN) [7]. Similarly, time-frequency analysis of vibrations have been used to diagnose wear in pump valve plates [8]. These analyses are effective at detecting issues such as cavitation, resonance and misaligned or warped components, and notifying operators when pump maintenance needs to take place.

However, pump vibration analysis is not (to the author's knowledge) used to monitor general system health and predict faults that occur outside of the pump itself. This is largely assessed using pipe vibrations [9], [10], which can provide insights into general system performance, or be used to locate discrete blockages downstream of pumps [11]. In the motivating scenario, access to the pipes is unavailable, and solder build-up does not form a localized blockage. Any solution needs to have low complexity so that it can be implemented on a low-cost microcontroller. Additionally, there is no pre-existing vibration data with which to establish baseline readings.

This paper uses Bayesian Change point Detection (BCD) to fulfill these requirements and assesses its suitability on a test bench. The use case fault can be presented as an unsupervised segmentation problem as the machine transitions from a functional to a non-functional state. BCD is particularly suitable for this type of problem [12]. It provides a measurement of the probability that, based on the collected information, a change in behaviour has occurred at a certain point [13], [14]. This can then be used to isolate the vibrational response of the pump at the point where the solder build-up is affecting the flow rate, or directly notify machine operators of the machine state. In rare cases where the deterioration of the pipe state is instantaneous rather than 
gradual, the change in vibrational response will still be able to inform machine operators of the need for maintenance. Successful applications in literature include the assessment of climate records to locate change points in climate regimes [14], detecting faults and failures in valves in an Unmanned Aerial Vehicle (UAV) fuel system [15], and detecting changes in the behaviour of a user of a text-messaging service [13]. BCD has also been used for online signal segmentation of epileptic brain activity [16] as well as activity recognition in a home [17]. The variety of applications demonstrates the flexibility of BCD. Alongside the number of change points, BCD can also provide an indication of the size of the changes [14], which can highlight significant events. The drawback of this approach is a sensitivity to the prior distribution assigned to the change points present in the system.

\section{Methodology}

The research methodology as illustrated in Fig.1 aims to use data analytics for improved process control in wave soldering. The data analytics approach is based on nondisruptive data extraction and processing using Bayesian Change point Detection. A test bench setup has been developed to replicate the wave-soldering machine behavior as the industrial machine and environment is not suitable for development. To keep it manageable in a laboratory environment, water is used instead of solder. The difference between the vibrational characteristics of water and solder should not affect the results of the BCD so long as the deterioration of the pipe leads to a change in measured vibrations that the BCD can detect. The experimental set-up and data collection are discussed in sections $\mathrm{A}$ and $\mathrm{B}$, and the $\mathrm{BCD}$ formulation is discussed in section $\mathrm{C}$.

\section{A. Test Bench Setup}

The test bench presented in this paper considers the behaviour of a pump moving water through a closed system. The flow rate is controlled by a gate valve, which is closed in discrete increments, from 0 (completely open) to 25 (completely closed). It mimics the effects of solder dross

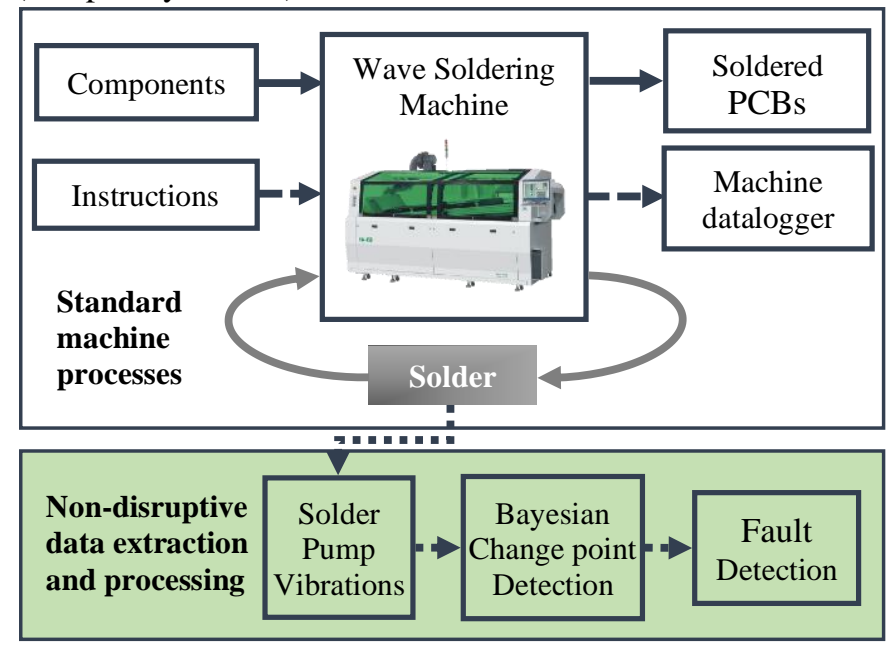

Figure 1. Wave Soldering Machine process diagram. Dotted arrows represent the movement of data. Solid arrows represent the movement of physical parts. The green box represents the non-disruptive paper contribution. build up in a wave soldering machine, which takes place over several weeks. As such, the steady state behaviour of the pump is the most relevant to this investigation. The schematic for this can be seen in Fig. 2, with a photo of the realised test bench in Fig. 3.

As the valve is closed, the change in the fluid's flow rate is monitored using the flow rate sensor YF-S201, connected to an Arduino. The vibration of the pump is recorded, using an accelerometer (LSM9DS1), at each increment to build up its response profile. This data is then processed at a Raspberry $\mathrm{Pi}$ $3 \mathrm{~B}$, and the results can be displayed in real time or uploaded to online networks, including any available IIoTs.

\section{B. Data Collection from the Test Bench}

The change in flowrate as the valve is closed is shown in Fig. 4. There is a small drop in the flow rate from states 0-16 before a transition period, and an almost linear drop to no flow from states 21-25. The minimal initial change is a result of the low flow rates used in this experiment, mimicking the low flow rates used in the wave-soldering machine. Pump vibrations are recorded with a sampling rate of $350 \mathrm{~Hz}$. At each valve state, 10 samples of 10,000 data points have been collected. The accelerometer has a range of $\pm 2 \mathrm{~g}$, a sensitivity

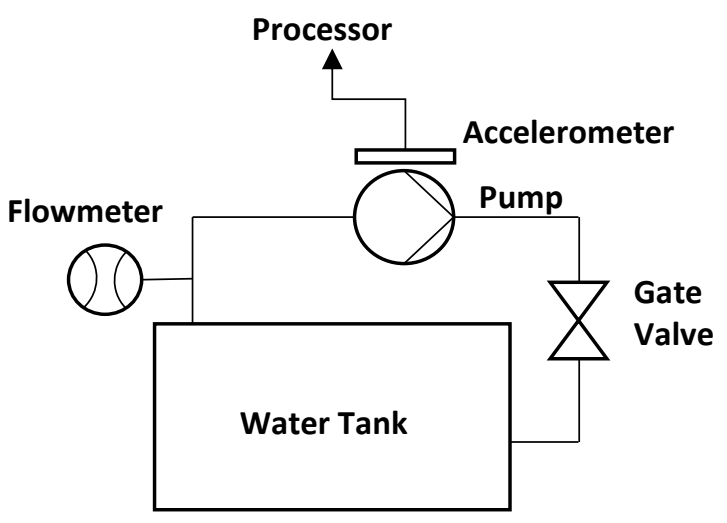

Figure 2. Test bench schematic

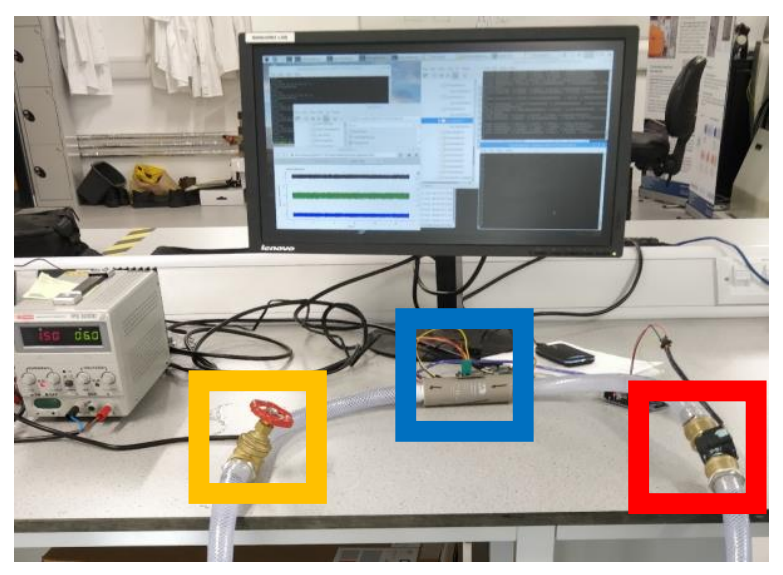

Figure 3. Test bench photo. Yellow: gate valve; blue: pump and accelerometer; red: flowmeter 
of $0.061 \mathrm{mg} / \mathrm{LSB}$, and collects samples in the $\mathrm{X}, \mathrm{Y}$ and $\mathrm{Z}$ axis. This is then converted into the absolute acceleration.

A fast Fourier transform (FFT) algorithm is used to extract the frequency and amplitude data from the vibrations [4]. Fig. 5 and Fig. 6 show the results of the FFT on the vibration data at valve states 0 and 25 respectively. The difference between the two is clearly visible, as the peak frequency shifts to the left. Additionally, it is clear that a lot of noise is present in the system - as would be present in an industrial setting. The solder in the use case might result in different vibrational characteristics. Despite this, the solder blockage should still result in a change in the recorded vibrations over time, which can be detected by the BCD algorithm. This is a significant benefit of the proposed methodology: as long as the input changes in a way that can be detected, the BCD algorithm will be able to assess the probability that a change point has occurred at a given point in time. This reduces the need for calibration procedures, which can be expensive and time consuming.

\section{Bayesian Change Point Detection}

Fig. 7 presents the pump data gathered by the accelerometer. Analysis of the time-domain vibration data has shown that it is not suitable for the analysis - it does not vary significantly enough across the different valve states to be a useful feature for predicting the current state. The mean of the amplitude of the time-domain data is shown to remain roughly constant throughout. However, the plotted features extracted from the FFT (peak frequency and maximum amplitude) can be seen to respond to the change in valve state. As such, these are the features used in the BCD algorithm presented in this paper.

Since both the peak frequency and the maximum amplitudes are used for the BCD in an identical fashion, the formulation is only shown for the maximum amplitude. The initial step requires choosing a model for the distribution of the collected data. This data can fall into three classifications: discrete, continuous and mixed. The chosen distribution has to be able to represent the data [11]. In this case, the amplitude (A) of the vibration data is continuous and samples are collected over the range of valve states, $V$, such that $A=$ i $\left.A_{1}, \ldots, A_{V}\right\}_{v=1, \ldots, V}$. It is safe to assume that any given sample for $A$ will have a value that falls within a normal distribution:

$$
A_{V} \sim N(\mu, \sigma)
$$

It is then assumed that over the range of valve states there is a single change point, $\tau$, during which $\mu$ changes to a different value - corresponding to the point where the mean amplitude changes and the pipe state deteriorates enough to significantly affect the flow rate. This leads to two $\mu$ parameters, one before $\tau$ and one for the rest of the remaining observation period:

$$
A_{V}=\left\{\begin{array}{l}
N\left(\mu_{1}, \sigma\right) \text { for } v<\tau \\
N\left(\mu_{2}, \sigma\right) \text { for } v \geq \tau
\end{array}\right.
$$

Since only the observed data is known, but no information about its distribution is available, the change point and probability distribution have to be inferred purely from the measurements. This can be expressed using Bayes' theorem:

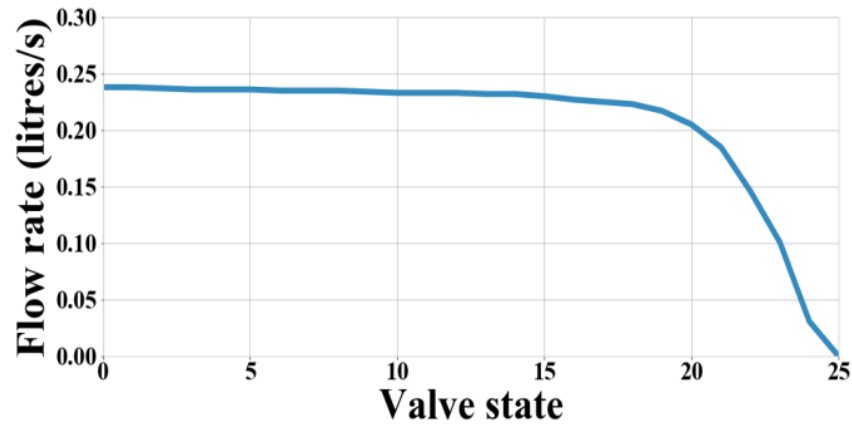

Figure 4. Flow rate against valve state

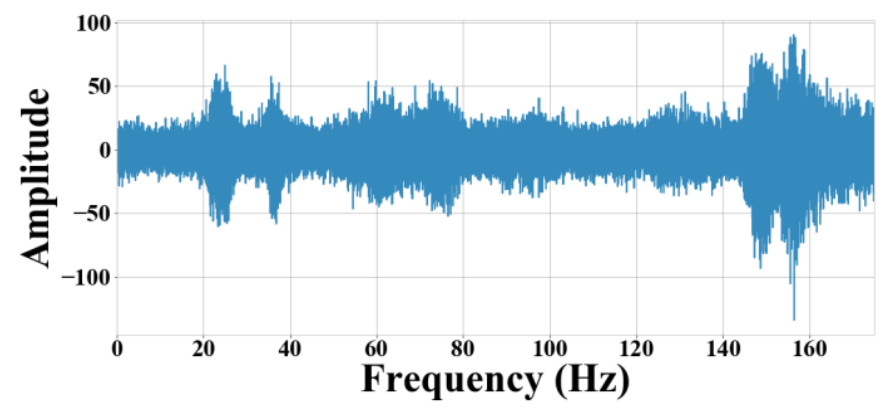

Figure 5. FFT of vibration data at state 0

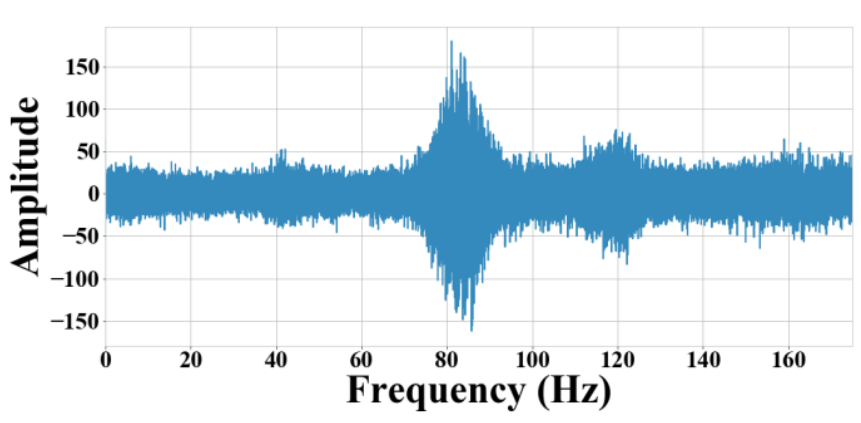

Figure 6. FFT of vibration data at state 25

$$
P\left(\mu_{1}, \mu_{2}, \sigma, \tau \mid A\right)=\frac{P\left(A \mid \mu_{1}, \mu_{2}, \sigma, \tau\right) P\left(\mu_{1}, \mu_{2}, \sigma, A\right)}{P(A)}
$$

An analytical solution to (3) can be found in [18], which marginilises the equation with respect to $\mu_{1}, \mu_{2}$, and $\sigma$ to provide an analytical solution for the posterior probability distribution $P(\tau \mid A)$. The posterior probability is a model parameter inferred by the observed data - in this case, the inferred probability that a change point $\tau$ occurred when the maximum amplitude had a value of $A$. However, in many practical cases a numerical method is employed to obtain this probability distribution because analytical solutions can become mathematically intractable [13]. This requires prior distributions to be assigned to the unknown parameters $P\left(\mu_{1}\right)$, $P\left(\mu_{2}\right), P(\sigma)$, and $P(\tau)$. Based on the observed data, the following prior distributions are assigned: $\mu_{1} \sim$ $\operatorname{Uniform}(100,200), \quad \mu_{2} \sim \operatorname{Uniform}(100,200), \quad \sigma \sim$ $\operatorname{Uniform}(0,100)$, and $\tau \sim$ Discrete, Uniform $(0,25)$. Since the actual values for $\mu_{1}, \mu_{2}$ and $\sigma$ are unknown, their prior distributions are assumed to be uniform over a range informed by the observed data. The value of $\tau$ is also unknown, but is 


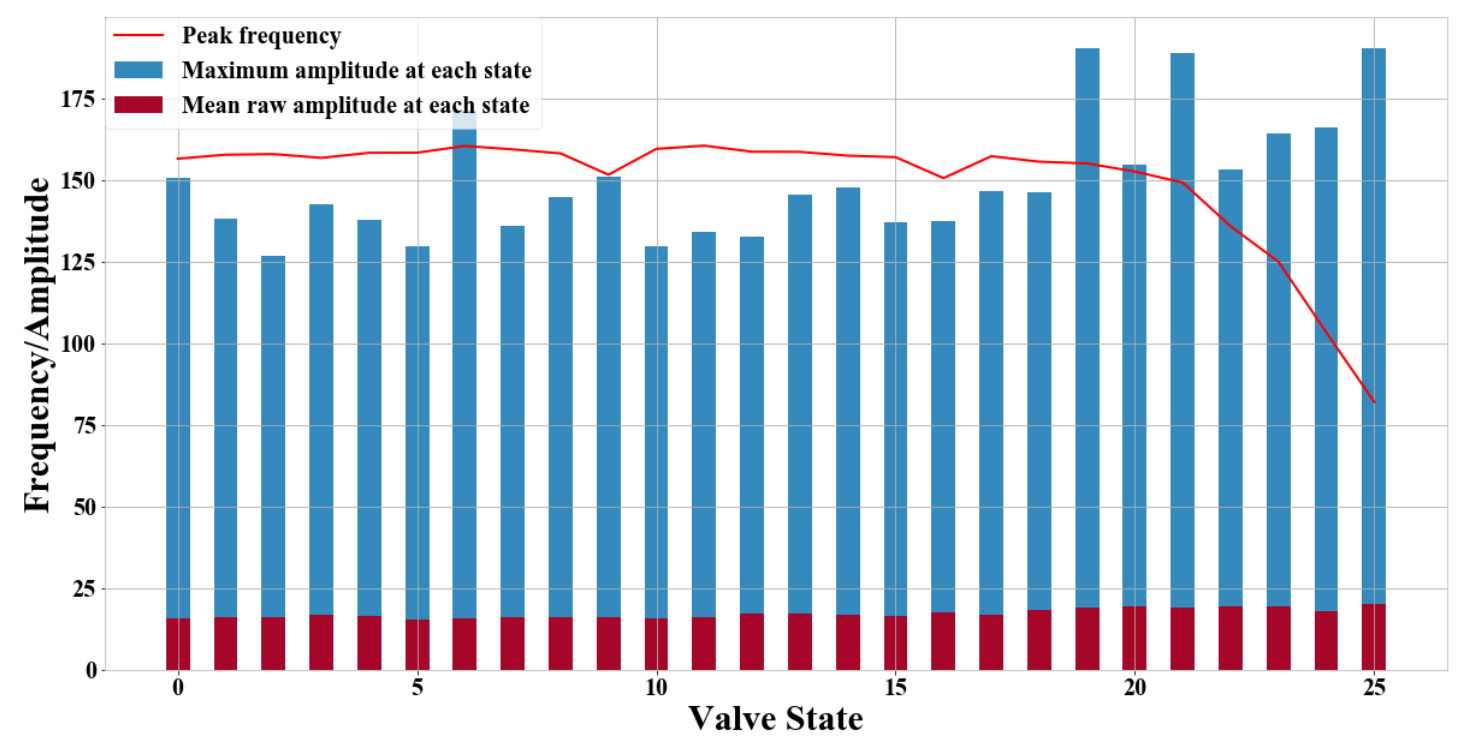

Figure 7. Data extraction from recorded pump vibrations at each valve state

known to be discrete as it has to be one of the tested valve states. Uniform distributions have been assigned to all the parameters to avoid biasing the BCD. However, other distributions can be used based on knowledge about the system in question.

Once the distributions have been assigned, this problem is solvable using readily available tools: in this case, PyMC3 is used because of its flexibility and power [13], [19]. It uses Markov Chain Monte Carlo (MCMC) methods, which generate samples from the given prior distributions and compares them to the existing data in order to generate the posterior distributions. In this case, the method used is adaptive slice sampling [20], chosen because of its suitability for sampling from discrete data distributions. Multiple MCMC chains are used to improve the quality of the results [21], with the first set of samples being discarded as part of the "burn-in period" which occurs before convergence. With this done, the distribution of $\tau, \mu_{1}, \mu_{2}$ and $\sigma$ can be plotted with simple functions of PyMC3. The plot of $\tau$ shows the likelihood of the change point occurring at any given valve state.

\section{RESUltS AND DISCUSSION}

From Fig. 4, it can be seen that flowrate reduction because of pressure drop is minimal until valve state 18 approximately $75 \%$ closed. After this point, the flow rate reduction appears to be linear.

Fig. 8 shows the plotted distributions of $\tau, \mu_{1}, \mu_{2}$ and $\sigma$ when applying the algorithm to the extracted maximum amplitudes. The higher value of $\mu_{2}$ over $\mu_{1}$ confirms that a change point has occurred and the amplitude of the pump vibrations increased as the valve was shut. The lack of an overlap between the values increases the certainty in the result [13]. The $\tau$ plot shows that the probability of the change point occurring is highest at valve state 19, although it does indicate a possibility of a change point as early as state 17 . This corresponds with the flow rate plot in Fig. 4. This information can then be plotted back onto the original amplitude reading to show the expected values based on the change point probabilities (Fig. 9).

Performing a similar analysis using the peak frequency gives the results plotted in Fig. 10. In this case $\mu_{1}$ is greater than $\mu_{2}$ as the frequency drops as the valve closes. $\tau$ indicates the change point is most likely to have occurred at valve state 23 , with lower chances of it being at state 22 and 24. Plotting this against the original frequency readings shows that it follows the frequency change very closely (Fig. 11).

This data analysis has shown that it is possible to use pump vibrations to assess the state of a system. It is possible to generate two different sources of data, which can then be used by the BCD algorithm to identify the points at which the flow rate begins to decrease. The predicted change points can be plotted against the recorded flow rate to demonstrate the effectiveness of the BCD based on the information it used (Fig. 12). Using the amplitude leads to earlier detection, which is valuable in situations where the processes involved are sensitive to any change. Using frequency leads to later detection, useful in processes which are less sensitive to changes and do not require immediate maintenance. In this case, the wave soldering machine chosen as a use case is sensitive to rapid changes in flow rate, so earlier detection is desirable.

\section{CONCLUSIONS}

This paper presents a new methodology for detecting an industrial fault in a wave-soldering machine. A BCD formulation is used to identify the point at which flowrate reductions happen as a result of the pressure drop caused by solder build-up in a pipe. It is assessed on a test bench analogous to a pump system in an industrial wave-soldering machine. A low-cost, non-disruptive monitoring device is used to collect pump vibration data to monitor machine states. The BCD then successfully generates the likelihood of a 


\section{Posterior Distribution of $\tau$}
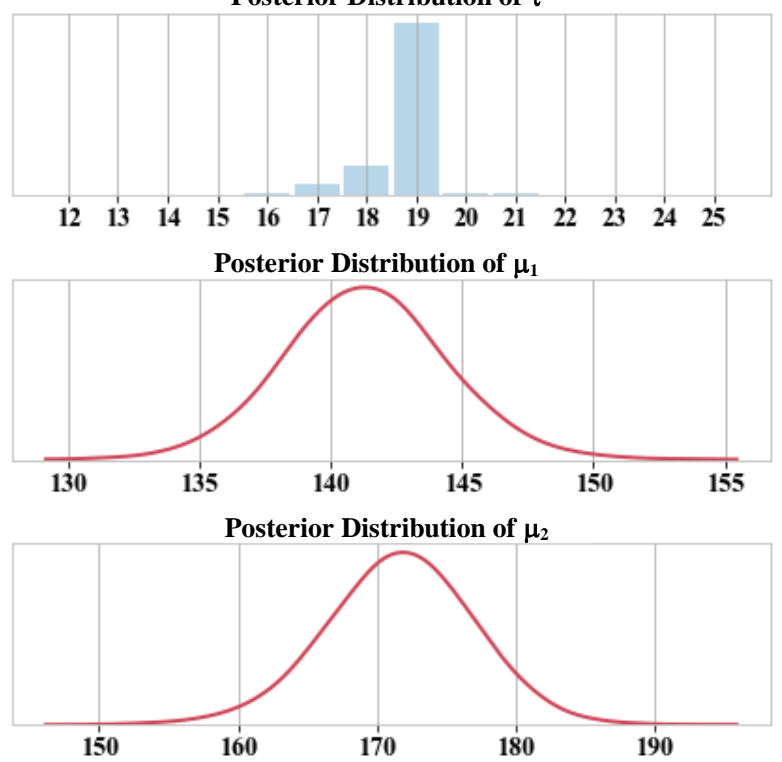

Posterior Distribution of $\sigma$

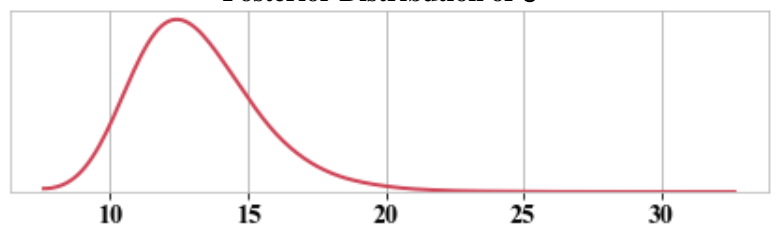

Figure 8. Posterior distribution of amplitude parameters

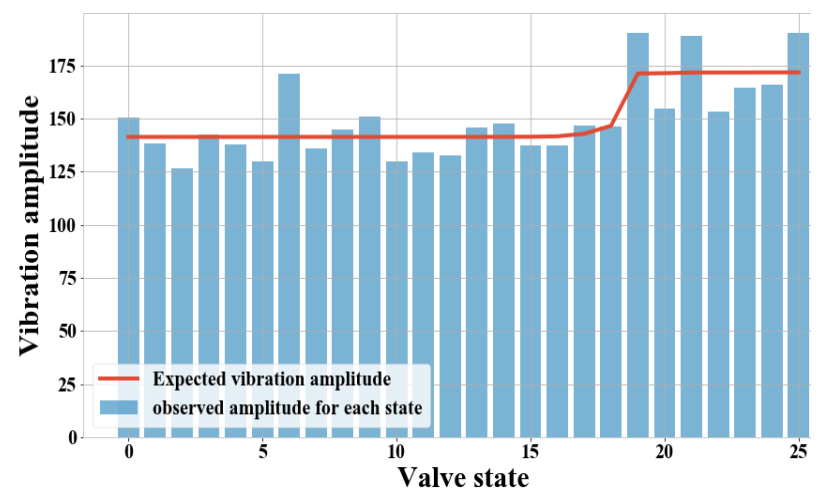

Figure 9. Expected amplitude value

change point occurring when the flow rate is reduced. This information can help machine operators better identify machine states and inform maintenance procedures.

Future work will use data collected directly from the wavesoldering machine, assessing the suitability of the chosen methods and the test bench with regards to actual industrial environments - especially with regards to industrial noise. Additionally, using an accelerometer capable of a faster sampling rate would help to identify whether the current setup is suitably sensitive or whether it needs to be improved. Adapting the algorithm to run online will also allow for live updates on IIoT and cloud platforms.
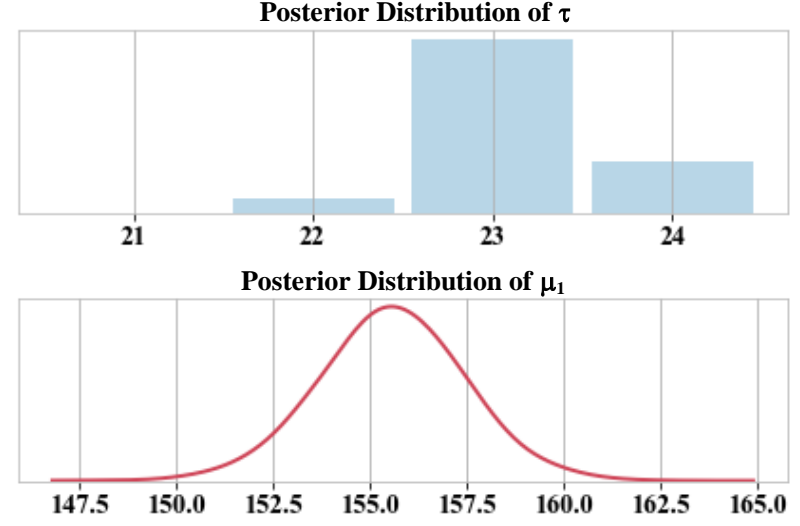

Posterior Distribution of $\mu_{2}$
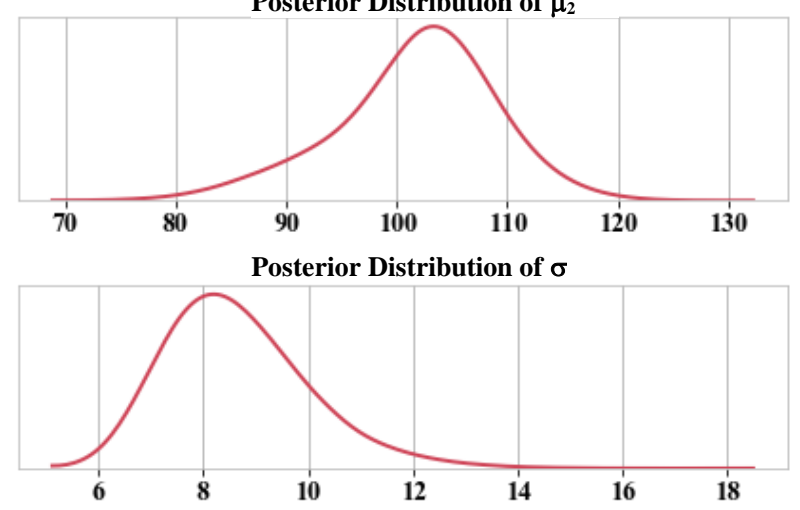

Figure 10 Posterior distribution of frequency parameters

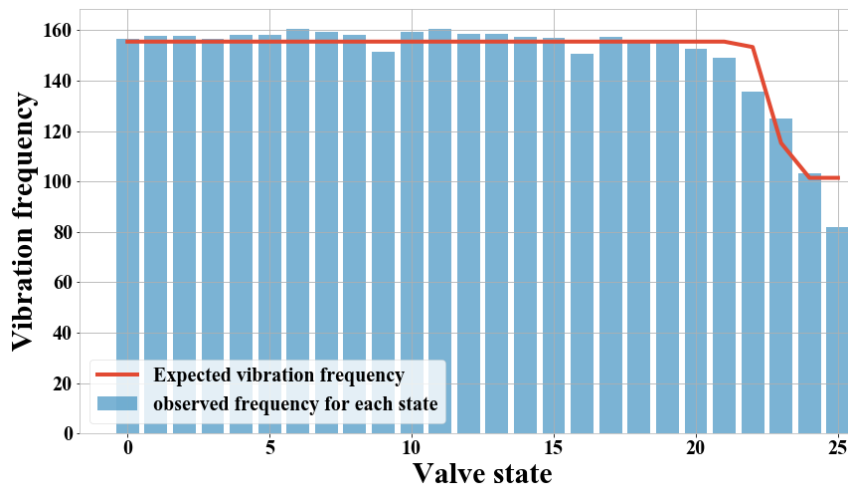

Figure 11. Expected frequency value

\section{ACKNOWLEDGMENT}

The authors would like to thank Siemens Congleton, especially Mr Carl German, for their helpful advice. This work is supported by the ESPRC iCASE entitled "Factory of the Future - Flexible, adaptable and self-optimised manufacturing systems".

\section{REFERENCES}

L. H. Chiang, E. L. Russell, and R. D. Braatz, Fault Detection and Diagnosis in Industrial Systems, 1st ed. London: Springer London, 2001.

[2] J. Manyika et al., "The Internet of Things: Mapping the value beyond the hype," McKinsey Glob. Inst., no. June, p. 144, 2015.

[3] J. Conway, "The Industrial Internet of Things: An Evolution to a Smart Manufacturing Enterprise," White Pap. Schneider Electr., 

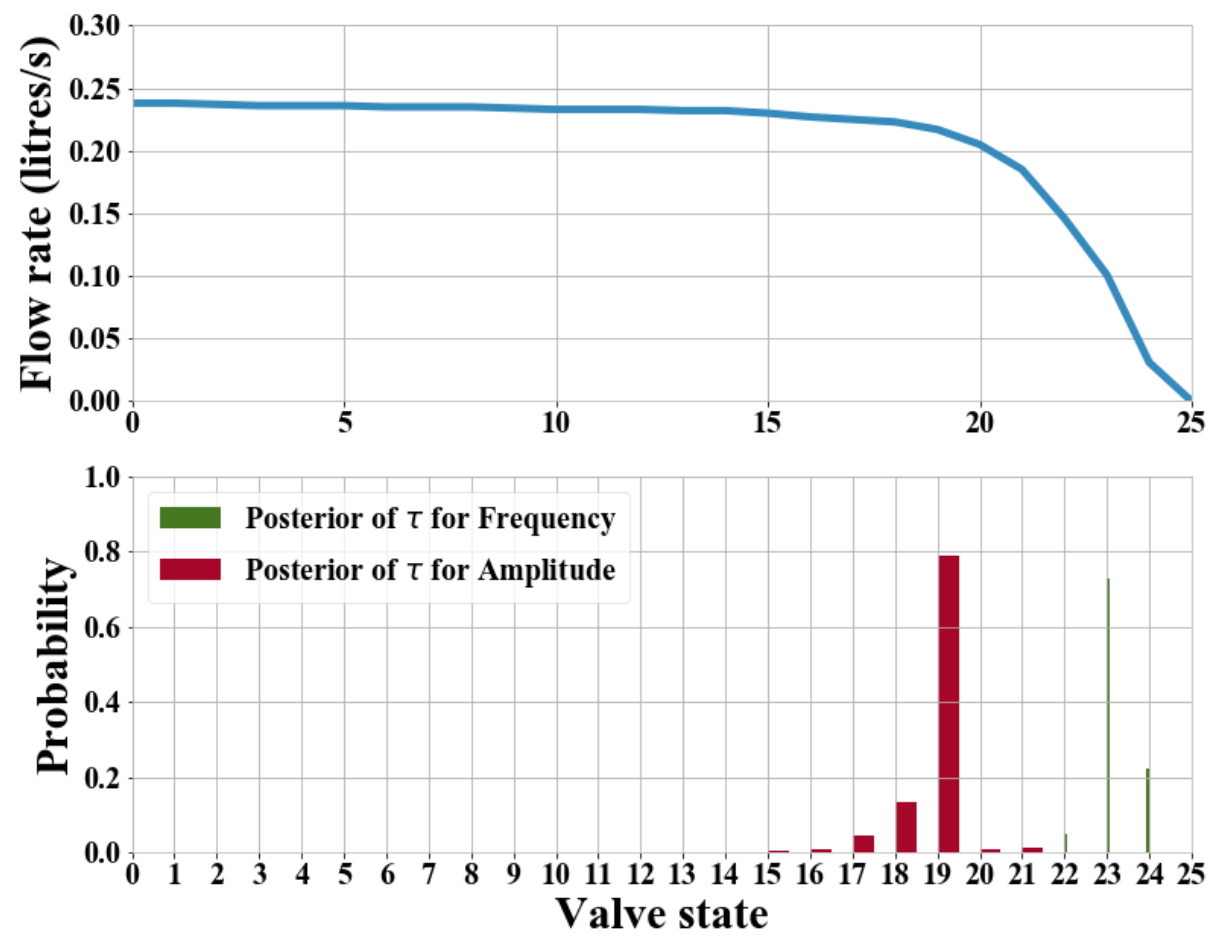

Figure 12. Flow rate and predicted changepoints against valve state

2016.

C. Scheffer, "Pump Condition Monitoring Through Vibration Analysis," PUMPS Maintenance, Des. Reliab. Conf. 2008 - IDC Technol., pp. 1-20, 2008.

R. Birajdar, R. Patil, and K. Khanzode, "Vibration and noise in centrifugal pump - sources and diagnosis methods," $3 r d$ Int. Conf. Integrity, Reliab. Fail., no. July, pp. 20-24, 2009.

D. L. Rivera, M. R. Scholz, M. Fritscher, M. Krauss, and K. Schilling, "Towards a Predictive Maintenance System of a Hydraulic Pump," 16th IFAC Symp. Inf. Control Probl. Manuf., 2018.

D. Siano and M. A. Panza, "Diagnostic method by using vibration analysis for pump fault detection," Energy Procedia, vol. 148, no. Ati, pp. 10-17, 2018.

J. Stojek, "Application of Time-Frequency Analysis for Diagnostics of Valve Plate Wear in Axial-Piston Pump," Arch. Mech. Eng., vol. 57, no. 3, pp. 309-322, 2010.

M. F. 9 9th Kim, Y.I., Simpson, A.R. and Lambert, "Behavior of orifices and blockages in unsteady pipe flows," no. January 2007, pp. 15-19, 2007.

A. S. A. COLLET and M. KÄLLMAN, Pipe Vibrations Measurement. Energiforsk, 2017.

N. L. T. Lile, M. H. M. Jaafar, M. R. Roslan, and M. S. M. Azmi, "Blockage Detection in Circular Pipe Using Vibration Analysis," Int. J. Adv. Sci. Eng. Inf. Technol., vol. 2, no. 3, p. 252, 2016.

A. Mohammad-Djafari and O. Féron, "A Bayesian approach to change points detection in time series," Int. J. Imaging Syst. Technol., vol. 16, no. 5, pp. 215-221, 2006.

C. Davidson-Pilon, Bayesian Methods for Hackers: Probabilistic Programming and Bayesian Inference (Addison-Wesley Data and Analytics), 1st ed. Addison-Wesley Professional, 2015.

E. Ruggieri, "A Bayesian approach to detecting change points in climatic records," Int. J. Climatol., vol. 33, no. 2, pp. 520-528, 2013.

O. Niculita, Z. Skaf, and I. K. Jennions, "The application of Bayesian change point detection in UAV fuel systems," in Procedia CIRP, 2014, vol. 22, no. 1, pp. 115-121.

R. Malladi, G. P. Kalamangalam, and B. Aazhang, "Online Bayesian change point detection algorithms for segmentation of epileptic activity," in Asilomar Conference on Signals, Systems and Computers, 2013, no. November 2013, pp. 1833-1837. S. Aminikhanghahi and D. J. Cook, "Using change point detection to automate daily activity segmentation," 2017 IEEE Int. Conf. Pervasive Comput. Commun. Work. PerCom Work. 2017, pp. 262-267, 2017.

[18] J. J. K. Ó Ruanaidh and W. J. Fitzgerald, Numerical Bayesian Methods Applied to Signal Processing. New York, NY: Springer New York, 1996.

[19] J. Salvatier, T. V. Wiecki, and C. Fonnesbeck, "Probabilistic programming in Python using PyMC3," PeerJ Comput. Sci., vol. 2016, no. 4, p. e55, Apr. 2016.

[20] R. M. Neal, "Slice sampling," Annals of Statistics, vol. 31, no. 3. pp. 705-767, 2003.

[21] A. Gelman and D. B. Rubin, "Inference from Iterative Simulation Using Multiple Sequences," Stat. Sci., vol. 7, no. 7, pp. 354-457$511,1992$. 